\title{
Coherent quantum oscillations and echo measurements of a Si charge qubit
}

\author{
Zhan Shi, C. B. Simmons, Daniel R. Ward, J. R. Prance, R. T. Mohr, Teck Seng Koh, John King Gamble, Xian Wu, \\ D. E. Savage, M. G. Lagally, Mark Friesen, S. N. Coppersmith, and M. A. Eriksson \\ University of Wisconsin-Madison, Madison, Wisconsin 53706, USA
}

(Received 4 March 2013; published 13 August 2013)

\begin{abstract}
Fast quantum oscillations of a charge qubit in a double quantum dot fabricated in a $\mathrm{Si} / \mathrm{SiGe}$ heterostructure are demonstrated and characterized experimentally. The measured inhomogeneous dephasing time $T_{2}^{*}$ ranges from $127 \mathrm{ps}$ to $2.1 \mathrm{~ns}$; it depends substantially on how the energy difference of the two qubit states varies with external voltages, consistent with a decoherence process that is dominated by detuning noise (charge noise that changes the asymmetry of the qubit's double-well potential). In the regime with the shortest $T_{2}^{*}$, applying a charge-echo pulse sequence increases the measured inhomogeneous decoherence time from 127 to $760 \mathrm{ps}$, demonstrating that low-frequency noise processes are an important dephasing mechanism.
\end{abstract}

DOI: 10.1103/PhysRevB.88.075416 PACS number(s): 73.63.Kv, 73.21.La, 73.23.Hk, 85.35.Gv

\section{INTRODUCTION}

Fast, coherent control of charge qubits has been demonstrated in both superconducting circuits ${ }^{1,2}$ and III-V semiconductor quantum dots. , $^{3,4}$ Beyond its intrinsic interest, understanding semiconductor charge qubit coherence is also important for spin qubits: mixing spin with charge degrees of freedom (either through spin-orbit coupling ${ }^{5}$ or the exchange interaction $^{6-11}$ ) enables faster spin manipulation than would otherwise be possible. When this mechanism is used, charge coherence can determine the ultimate fidelity of a spin qubit. ${ }^{11}$

Here, we present experimental measurements of fast coherent quantum oscillations between the $(2,1)$ and $(1,2)$ charge states of a qubit formed in a $\mathrm{Si} / \mathrm{SiGe}$ double quantum dot. Given the Bloch sphere ${ }^{12}$ with the $\pm z$ axes representing the $(1,2)$ and $(2,1)$ states, rotations about the $x$ axis, or Larmor oscillations, are observed when the $(2,1)$ and $(1,2)$ states are energetically degenerate, with a decoherence time $T_{2}^{*}=2.1 \mathrm{~ns}$. Rotations about the $z$ axis are probed in the Ramsey fringe experiment and are observed with a shorter coherence time, $T_{2}^{*}=127 \mathrm{ps}$. The Ramsey fringes are measured when the charge qubit is operated in a regime where the energy difference between the qubit states depends strongly on detuning $\varepsilon$, the energy difference between the $(2,1)$ and $(1,2)$ charge states, and so is highly sensitive to charge noise in the local environment. The significantly different coherence times are consistent with the dominant dephasing mechanism arising from fluctuations in $\varepsilon$, as has previously been observed in GaAs devices. ${ }^{4} \mathrm{We}$ also present measurements of charge echo in a semiconductor quantum dot charge qubit, with the echo sequence yielding an increase of the coherence time from 127 to $760 \mathrm{ps}$. The results demonstrate control of a silicon charge qubit, and they show that charge echo can be exploited to improve its coherence.

\section{EXPERIMENT}

The device measured in the experiment was fabricated in a Si/SiGe heterostructure as described in Refs. 13 and 14; a scanning electron microscope image of an identical device is shown in Fig. 1(a). The quantum point contact on the right side of the double dot is used as a charge sensor. Using magnetospectroscopy measurements, ${ }^{15}$ we confirm the valence charge occupation of the double dot is $(2,1)$ or $(1,2)$; either or both dots may contain a closed shell beneath the valence electrons, although if present such shells do not appear to play a role in the work we report.

\section{A. Larmor oscillations ( $x$ rotations)}

Figure 1(b) shows an energy level diagram of the anticrossing between $(2,1)$ and $(1,2)$ as a function of detuning energy $\varepsilon$. Near the charge degeneracy point $(\varepsilon=0)$ the system is well-described by the Hamiltonian of a two-state system:

$$
H=\left(\begin{array}{cc}
\varepsilon / 2 & \Delta \\
\Delta & -\varepsilon / 2
\end{array}\right) .
$$

Coherent oscillations between the two charge states can be observed when the detuning $\varepsilon$ is changed abruptly. For example, starting in a position eigenstate with charge occupation $(2,1)$ at large negative $\varepsilon$, after increasing the detuning suddenly to $\varepsilon=0$, as shown by the red horizontal arrow in Fig. 1(b), the system Hamiltonian becomes $H=\Delta \sigma_{x}$, where $\sigma_{x}$ is the usual Pauli matrix. Subsequently, the system oscillates between $(2,1)$ and $(1,2)$ at the Larmor angular frequency $2 \Delta / \hbar$. More generally, nonadiabatically increasing the detuning $\varepsilon$ to a value $\varepsilon^{\prime}$ is expected to induce oscillations at the angular frequency $\Omega_{\mathrm{R}}=\sqrt{\varepsilon^{\prime 2}+4 \Delta^{2}} / \hbar$ about a tilted axis: as one moves away from the polarization line at which $\varepsilon=0$, the oscillations increase in frequency and decrease in amplitude.

Figure 1(c) shows a number of Larmor oscillations between the $(2,1)$ and $(1,2)$ charge states. Square pulses of duration $t_{\mathrm{p}}$ and amplitude $V_{\mathrm{p}}=800 \mathrm{mV}$ are applied to gate $\mathrm{L}$ at frequency $40 \mathrm{MHz}$. The transconductance $G_{\mathrm{L}}=\partial I_{\mathrm{QPC}} / \partial V_{\mathrm{L}}$ is plotted as a function of $t_{\mathrm{p}}$ and $\varepsilon_{\mathrm{p}}$, the position in detuning of the peak of the pulse. The connection between $V_{\mathrm{L}}, t_{\mathrm{p}}, \varepsilon_{\mathrm{p}}$, and other details can be found in Appendix A. Oscillations of the signal are apparent out to more than three nanoseconds.

To enable comparison to theory, and to obtain quantitative dephasing times from the experiment, we integrate the data presented in Fig. 1(c) and extract the probability $P_{(1,2)}$ of occupying the $(1,2)$ charge state (see Appendix A for details). Figure 1(e) presents the charge oscillation near zero detuning from the integrated data of (c) as the solid red trace. By fitting the amplitude of the oscillations to exponential decays, we extract a dephasing time $T_{2}^{*}=2.1 \pm 0.4$ ns near $\varepsilon_{\mathrm{p}}=0$, 

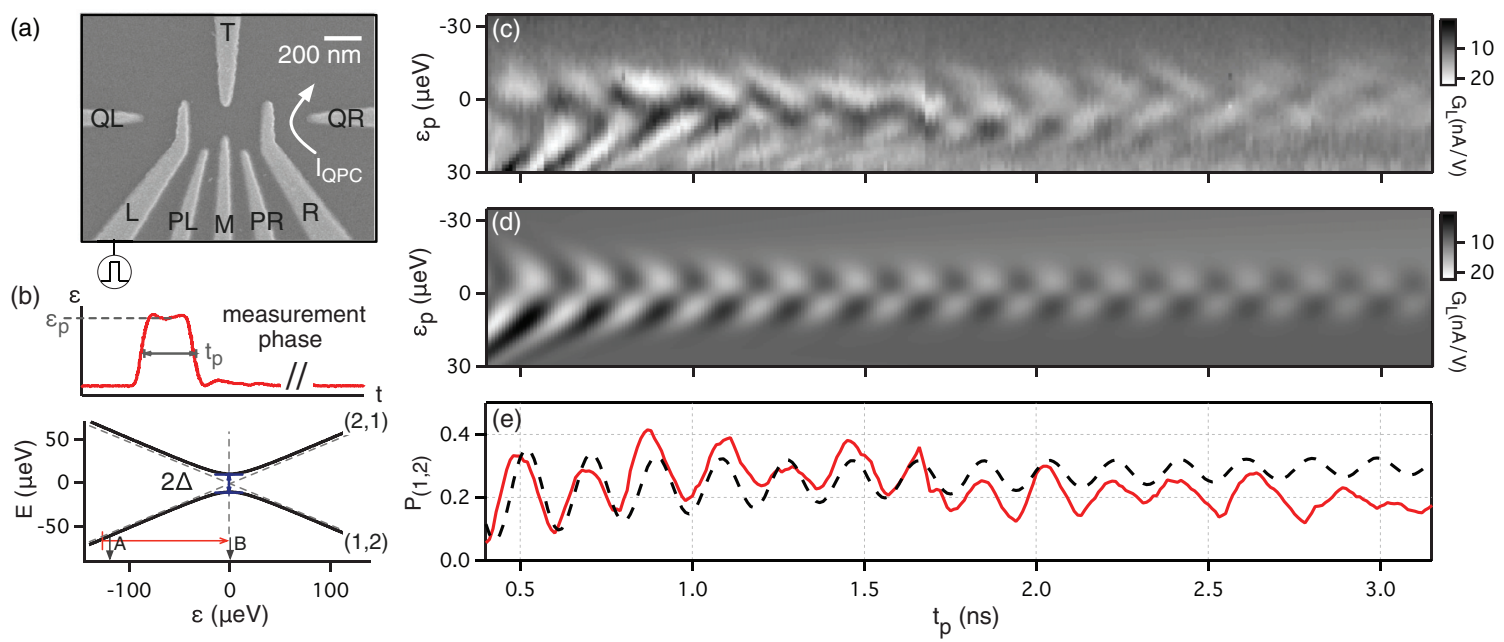

FIG. 1. (Color online) Larmor oscillations. (a) SEM image of a device identical to the one used in the experiment. The current $I_{\mathrm{QPC}}$ is used for charge sensing through a measurement of its transconductance $G_{\mathrm{L}}=\partial I_{\mathrm{QPC}} / \partial V_{\mathrm{L}}$, and voltage pulses are applied to gate L. (b) (Bottom) Diagram of energy levels vs detuning $\varepsilon$, showing the anticrossing between $(2,1)$ and $(1,2)$ charge configurations. The red arrow represents an applied voltage pulse with the dc level and amplitude of the pulse chosen to place the peak of the pulse at $\varepsilon=0$. (Top) Example pulse showing the pulse peak position $\varepsilon_{\mathrm{p}}$, pulse duration $t_{\mathrm{p}}$, and the measurement phase. (c) Larmor oscillations between the $(2,1)$ and $(1,2)$ charge configurations, as a function of pulse duration $t_{\mathrm{p}}$ and pulse peak position $\varepsilon_{\mathrm{p}}$. The Larmor oscillations that reflect rotations between the states with $(2,1)$ and $(1,2)$ charge occupations are manifest near $\varepsilon_{\mathrm{p}}=0$. (d) Numerical simulation of the Larmor oscillations of (c), using an 80-ps-pulse rise time and the energy level diagram in (b) with best fit parameter $\Delta=10.8 \mu \mathrm{eV}(\Delta / h=2.62 \mathrm{GHz})$. (e) Solid red: a horizontal cut taken near zero detuning from the integrated data of (c), showing Larmor oscillations as a function of pulse duration. Dashed black: a corresponding cut from the simulated charge occupation data.

marked by label B in Fig. 1(b). This dephasing time is relatively long, because at the anticrossing the difference in energy between the eigenstates is insensitive to detuning fluctuations.

Numerical simulations of the experiment were performed based on the energy level diagram in Fig. 1(b). We model the dynamical evolution of the density matrix $\rho$ of the system as a function of detuning $\varepsilon_{\mathrm{p}}$ and pulse duration $t_{\mathrm{p}}$ using a master equation: ${ }^{12,16}$

$$
\dot{\rho}=-\frac{i}{\hbar}[H, \rho]+D,
$$

where $D$ is a phenomenological term that describes pure dephasing of the charge state under the assumption of Markovian dynamics. $D$ is given, in the $\{|(2,1)\rangle,|(1,2)\rangle\}$ basis, by

$$
D=-\left(\begin{array}{cc}
0 & \Gamma_{0} \rho_{12} \\
\Gamma_{0} \rho_{21} & 0
\end{array}\right),
$$

where $\Gamma_{0}=0.48 \mathrm{GHz}$ is the dephasing rate $\left(1 / T_{2}^{*}\right)$ measured near zero detuning. This dephasing can have contributions both from the effect of charge noise on the tunnel coupling and from second-order effects from detuning noise, since the qubit is to first order insensitive to detuning noise at the charge degeneracy point. The $(2,1)$ and $(1,2)$ occupation probabilities are extracted at the end of the $t_{\mathrm{p}}$ pulse and, for the duration of the measurement phase, are allowed to relax exponentially to the ground state $(2,1)$ with a relaxation time $T_{1}$. The simulated charge occupation is determined by averaging the charge state for the entire $25 \mathrm{~ns}$ pulse period. Low-frequency fluctuations in the detuning $\varepsilon$ are incorporated following Ref. 4, by performing a convolution of the results at each $\varepsilon$ with a Gaussian in $\varepsilon$ of width $\sigma_{\varepsilon}=5 \mu \mathrm{eV}$. The best fit to the data is found with a charge relaxation time $T_{1}=18 \mathrm{~ns}$. The results obtained using the tunneling amplitude $\Delta=10.8 \mu \mathrm{eV}$ $(\Delta / h=2.62 \mathrm{GHz})$, shown in Fig. $1(\mathrm{~d})$, agree well with the data. Figure 1(e) is a horizontal cut showing the measured and simulated charge occupation data near $\varepsilon=0$. Again, good agreement between the data and the calculation is found.

\section{B. Ramsey fringes ( $z$ rotations)}

We now demonstrate coherent rotations of the charge qubit about the $z$ axis of the Bloch sphere by performing a Ramsey fringe experiment, ${ }^{17,18}$ using the two-pulse sequence shown in the inset to Fig. 2(a). Starting at a negative detuning in the $(2,1)$ state, the qubit is pulsed to the $(2,1)-(1,2)$ anticrossing, which causes the Bloch vector to rotate around the $x$-axis. The duration of this first pulse is chosen [based on the data in Fig. 1(c)] so that the Bloch vector is rotated around the $x$ axis by a nominal angle of $5 \pi / 2$, taking it from being along $z$ to being in the $x-y$ plane (we use a $5 \pi / 2$ pulse of amplitude $600 \mathrm{mV}$ and duration $280 \mathrm{ps}$ instead of a $\pi / 2$ pulse, because of the difficulty of applying high-quality pulses shorter than $100 \mathrm{ps}$ ). After a variable free evolution time $\tau$ at the base level of the detuning $\varepsilon_{\mathrm{b}}$, during which the Bloch vector rotates about the $z$ axis, a second pulse is applied to rotate the state about the $x$ axis on the Bloch sphere by another $5 \pi / 2$. The charge measured at the end of this process oscillates as a function of the time $\tau$ between the two pulses at a frequency determined by the difference in energy of the states involved at the base level of detuning.

Figure 2(a) shows the transconductance $G_{\mathrm{L}}$ of the charge sensor as a function of the base level detuning $\varepsilon_{\mathrm{b}}$ and the 

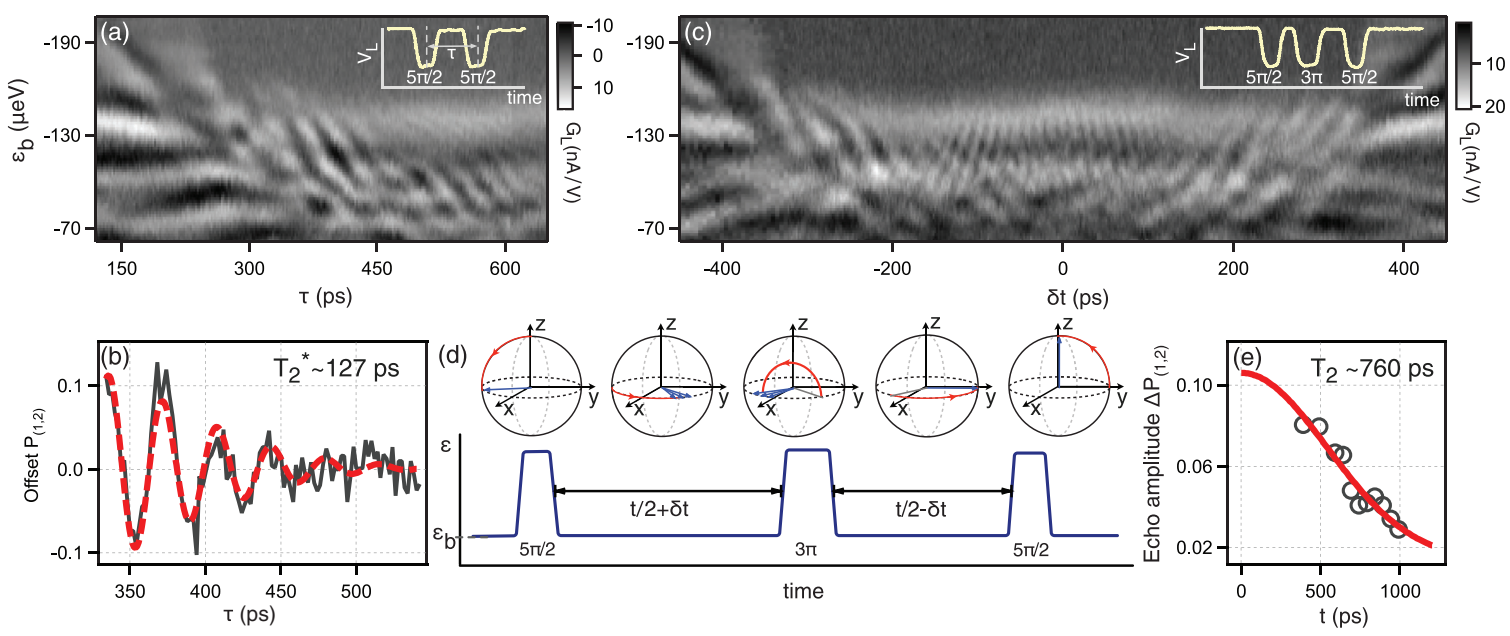

FIG. 2. (Color online) (a) Ramsey fringes: QPC transconductance $G_{\mathrm{L}}$ as a function of the base level of detuning $\varepsilon_{\mathrm{b}}$ and the time $\tau$ between two $5 \pi / 2$ pulses, as shown in the inset. The oscillations observed in the region where $\tau>280 \mathrm{ps}$ reflect the rotation of the Bloch vector around the $z$ axis in the $x-y$ plane. (b) Gray: a line cut of the integrated data of (a) after removal of a smooth background, as described in Appendix A. Red: fit to the form $A \exp \left[-\left(\tau-\tau_{0}\right)^{2} / T_{2}^{* 2}\right] \cos (\omega t+\phi)+C$, which yields $T_{2}^{*}=127 \pm 8$ ps. (c) Charge echo: QPC transconductance $G_{\mathrm{L}}$ as a function of $\varepsilon_{\mathrm{b}}$ and $\delta t$ for $t=640 \mathrm{ps}$. Oscillations are strongest near $\delta t=0$, where equal free evolution times before and after the $3 \pi x$ rotation provide the best correction for slow inhomogeneous dephasing. Away from $\delta t=0$, increasingly mismatched free evolution times provide less correction, and the oscillations decay with characteristic time $T_{2}^{*}$. (Inset) Trace of the pulse sequence used to acquire these data. (d) Sequence of pulses used to implement charge echo: a nominal $5 \pi / 2 x$ rotation into the $x$ - $y$ plane, free evolution for a time $t / 2+\delta t$, a $3 \pi x$ rotation, free evolution time $t / 2-\delta t$, and a $5 \pi / 2 x$ rotation out of the $x-y$ plane. For this experiment, $5 \pi / 2$ pulses have a duration of 280 ps and $3 \pi$ pulses have a duration of $330 \mathrm{ps}$. (e) Dark circles: charge oscillation amplitudes $\Delta P_{(1,2)}$ as a function of the free evolution time $t$, extracted from data sets with different $t$ values. The echo amplitude decays as $t$ increases. A fit of the decay to the Gaussian form $y_{0}+A \exp \left[-\left(t / T_{2}\right)^{2}\right]$ yields $T_{2}=760 \pm 190 \mathrm{ps}$, significantly longer than $T_{2}^{*}$. Thus the echo pulse sequence corrects for slow inhomogeneous dephasing and extends the coherence time.

time $\tau$, in the presence of the two-pulse pattern applied at a repetition rate of $25 \mathrm{MHz}$. For very short $\tau$, the $5 \pi / 2$ pulses overlap and one is essentially performing a Larmor oscillation experiment. At $\tau \gtrsim 280 \mathrm{ps}$, the time interval between the end of the first $5 \pi / 2$ pulse and the start of the second becomes nonzero, and the observed oscillations correspond to a Ramsey fringe measurement.

To analyze these data quantitatively, we again integrate the data and extract $P_{(1,2)}$. Figure 2(b) shows a cut through the integrated data at the value of detuning $\varepsilon_{\mathrm{b}}=-120 \mu \mathrm{eV}$ marked by the arrow labeled A in Fig. 1(b), after subtraction of a smooth background (see Appendix A). These Ramsey fringes oscillate at $28 \mathrm{GHz}$, which agrees with the energy difference of the two charge states at that detuning value. We fit these oscillations to the product of a cosine function and a Gaussian. ${ }^{4}$ This procedure yields $T_{2}^{*}=127 \pm 8 \mathrm{ps}$, much shorter than $T_{2}^{*}=2.1 \mathrm{~ns}$ measured in the Larmor experiment. As is clear from Fig. 1(b), at the large negative detuning where the oscillations are being generated, the energy levels diverge rapidly from each other as a function of $\varepsilon$, providing no protection from charge noise. The dephasing time in this Ramsey fringe experiment is nonetheless twice as long as the value of 60 ps obtained by measuring Ramsey fringes for a GaAs charge qubit and using a similar fitting procedure to extract $T_{2}^{*} \cdot{ }^{18}$ Calculations following the methods of Ref. 19 show that the charge dephasing rate in GaAs from polar optical phonons may be of order $1 \mathrm{GHz}$, whereas similar calculations for phonon-induced charge dephasing in $\mathrm{Si}$ yield values of order $0.5 \mathrm{MHz}$. Thus, in both materials, and particularly in $\mathrm{Si}$, improvements may be possible through a reduction of excess charge noise.

\section{Charge-echo experiment}

We now demonstrate that the effects of inhomogeneous dephasing can be ameliorated using a charge-echo method. ${ }^{20}$ Charge echo is implemented by applying the voltage pulse sequence shown in Fig. 2(d). When the tips of the pulses reach the $(2,1)-(1,2)$ anticrossing, the pulse sequence consists of a $5 \pi / 2$ pulse (which rotates the Bloch vector into the $x-y$ plane), a free evolution at the base detuning for a time $t / 2+\delta t$, a $3 \pi$ pulse (which flips the Bloch vector to its mirror image with respect to $x-z$ plane), a second free evolution at the base detuning for a time $t / 2-\delta t$, and a second $5 \pi / 2$ pulse (which rotates the Bloch vector about the $x$ axis again). Figure 2(c) shows the transconductance measured as a function of the detuning $\varepsilon_{\mathrm{b}}$ and the time $\delta t$ for $t=640 \mathrm{ps}$. The oscillations are strongest around $\delta t=0$, where the echo sequence best corrects for inhomogeneous dephasing. As $|\delta t|$ increases, more time is spent performing an uncorrected $z$-rotation, and the oscillation amplitude decays with characteristic time $T_{2}^{*}$, just as in the Ramsey fringe experiment. As the total free evolution time $t$ increases, the oscillation amplitude will decay with characteristic time $T_{2}$. To extract $T_{2}$, we perform the echo pulse sequence for multiple values of $t$. We convert the transconductance data to charge occupation data and extract the amplitude of the charge oscillation $\Delta P_{(1,2)}$ (see Appendix B). Figure 2(e) shows the extracted value of $\Delta P_{(1,2)}$ 
for each data set, plotted as a function of $t$. The echo amplitude clearly decays as $t$ is made longer. A Gaussian fit of the decay yields $T_{2}=760 \pm 190 \mathrm{ps}$. The significant increase in coherence time indicates that low-frequency noise processes play an important role in limiting qubit coherence.

\section{CONCLUSION}

In summary, we have observed coherent quantum charge oscillations in a qubit formed in a $\mathrm{Si} / \mathrm{SiGe}$ double quantum dot. The coherence time $T_{2}^{*}$ is $2.1 \mathrm{~ns}$ for Larmor oscillations or $x$ rotations, at the charge degeneracy point, and is $127 \mathrm{ps}$ for Ramsey fringes that reflect rotations about the $z$ axis of the Bloch sphere. Implementation of a charge-echo pulse sequence increases the decoherence time from 127 to $760 \mathrm{ps}$ in the regime where the energy difference between the two qubit states depends substantially on detuning.

\section{ACKNOWLEDGMENTS}

We thank Sankar Das Sarma and Jason Petta for useful discussions. This research was supported in part by the US Army Research Office (W911NF-08-1-0482, W911NF-12-10607), the NSF (DMR-0805045), and by the United States Department of Defense. The views and conclusions contained in this document are those of the authors and should not be interpreted as representing the official policies, either expressly or implied, of the US Government. This research utilized NSFsupported shared facilities at the University of WisconsinMadison.

\section{APPENDIX A: MEASUREMENT DETAILS AND LINE CUTS OF LARMOR AND RAMSEY OSCILLATIONS}

The data shown in Fig. 1(c) of the main text are acquired by sweeping the voltage on gate $\mathrm{L}$ and the pulse duration $t_{\mathrm{p}}$. As $t_{\mathrm{p}}$ increases, because of the high-pass filter in the bias-tee for the high-frequency line, the time-averaged voltage on gate $\mathrm{L}$ changes, resulting in a linear change in the relationship between $V_{\mathrm{L}}$ and detuning $\varepsilon$. Thus, to remove this offset, before converting $V_{\mathrm{L}}$ to $\varepsilon$, we shift each vertical scan line by an amount $\delta V_{\mathrm{L}}=V_{\mathrm{p}} \times t_{\mathrm{p}} \times f_{\text {rep }}$, where $V_{\mathrm{p}}$ is the pulse amplitude and $f_{\text {rep }}$ is the pulse repetition frequency. The vertical axis in Fig. 1(c) then is converted to $\varepsilon_{\mathrm{p}}$, the detuning value at the peak of the pulse, by noting that the main, slowest oscillation corresponds to $\varepsilon_{\mathrm{p}}=0$, and by fitting the change in Larmor frequency as a function of $V_{\mathrm{L}}$ for positive $\varepsilon_{\mathrm{p}}$. For our experimental conditions, $1 \mathrm{~V}$ applied to the high-frequency coaxial line connected to gate $\mathrm{L}$ results in a change in gate voltage of $6.6 \mathrm{mV}$ on that gate.

To enable direct comparison between the simulation and the data, we convert the simulated charge occupation to QPC current using the measured QPC sensitivity of $18 \mathrm{pA} /$ electron and the measured cross talk between $V_{\mathrm{L}}$ and the QPC current $8.87 \mathrm{nA} / \mathrm{V}$. We then differentiate the current with respect to $V_{\mathrm{L}}$ to produce simulated transconductance data in Fig. 1(d).

The connection between $V_{\mathrm{L}}$ and detuning $\varepsilon$ is $\alpha_{\mathrm{L}, \varepsilon}=$ $-24 \mu \mathrm{eV} / \mathrm{mV}$ for the Larmor oscillation data and $\alpha_{\mathrm{L}, \varepsilon}=$ $-36 \mu \mathrm{eV} / \mathrm{mV}$ for the Ramsey fringe and charge-echo data (these data sets were acquired at different gate voltage tunings
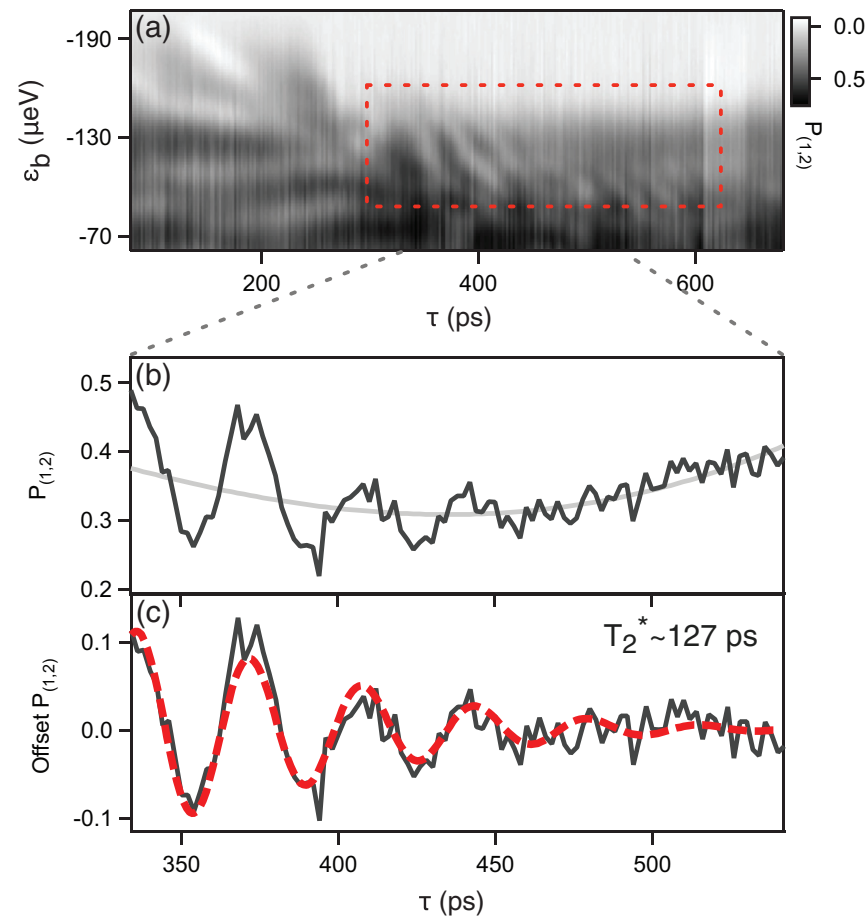

FIG. 3. (Color online) Ramsey fringe analysis. (a) Integration of the transconductance $G_{\mathrm{L}}$ from Fig. 2(a) of the main text yields the probability $P_{(1,2)}$ of occupying the $(1,2)$ charge state in the regime where the $(2,1)$ charge state is the ground state. The data are normalized by noting that the total charge transferred across the polarization line is one electron. The red dashed box indicates the location of the fringes. (b) Dark gray: line cut of the data in (a), at the detuning $\varepsilon=-120 \mu \mathrm{eV}$. Light gray: Smooth background that is subtracted from the line cut before fitting the data to a damped sinusoidal form. (c) [The same as Fig. 2(b) in the main text.] Gray: the data from (b) after subtraction of the smooth background. Red: fit to the form $A \exp \left[-\left(\tau-\tau_{0}\right)^{2} / T_{2}^{* 2}\right] \cos (\omega t+\phi)+C$, which yields $T_{2}^{*}=127 \pm 8$ ps.

of the dots). The lever arm is determined by fitting the Larmor oscillation frequency as a function of gate voltage $f=\sqrt{\alpha_{\mathrm{L}, \varepsilon} V_{L}^{2}+4 \Delta^{2}} / h$.

Figure 3(a) shows the results of the integration of the data in Fig. 2(a) in the main text, normalized to obtain the probability $P_{(1,2)}$ of being in the $(1,2)$ charge configuration when $(2,1)$ is the ground state. Figure 3(b) shows a line cut through the plot in Fig. 3(a) at the value of $\varepsilon=-120 \mu \mathrm{eV}$. Figure 3(c), which is the same as Fig. 2(c) in the main text, shows the Ramsey fringes after subtraction of the smooth background shown in Fig. 3(b).

\section{APPENDIX B: ANALYSIS OF CHARGE ECHO EXPERIMENT}

To extract $T_{2}$ from the charge-echo data, we perform two analyses. In the first, the oscillation amplitude is quantified at a given value of the detuning by analyzing the fast Fourier transform (FFT) of the probability $P_{(1,2)}$. In the second, the oscillation amplitude is quantified at a given oscillation frequency by analyzing the FFT of $G_{\mathrm{L}}$. As shown below, the results from the two methods are consistent. 

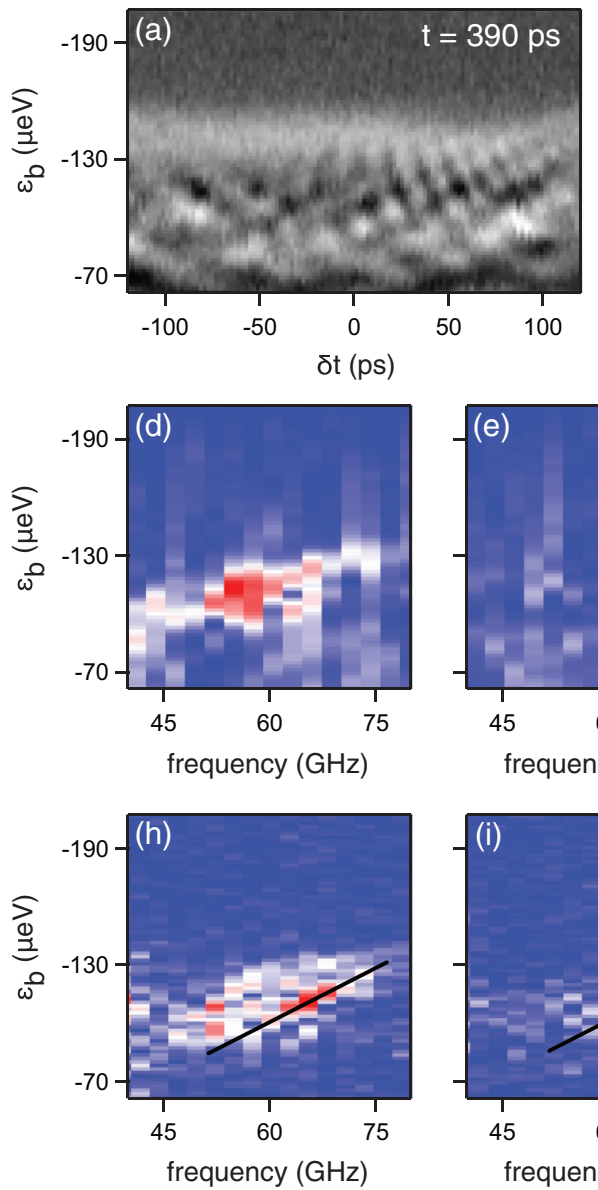

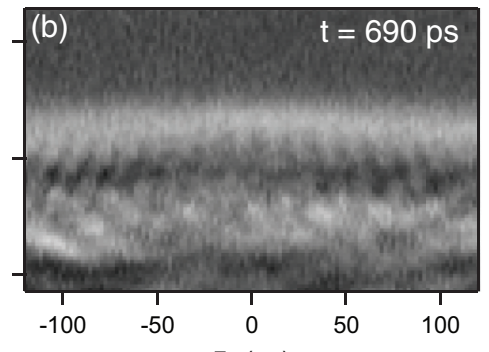

$\delta t(p s)$
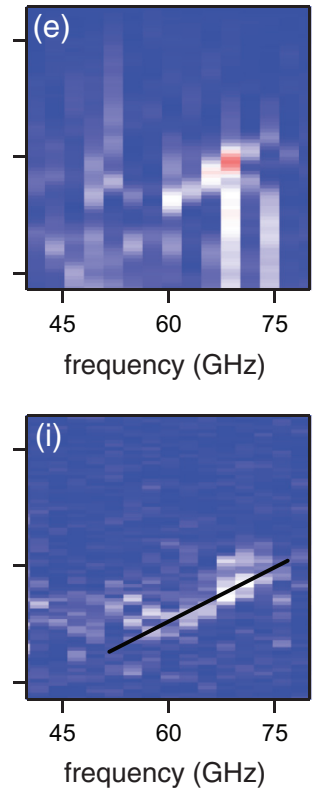
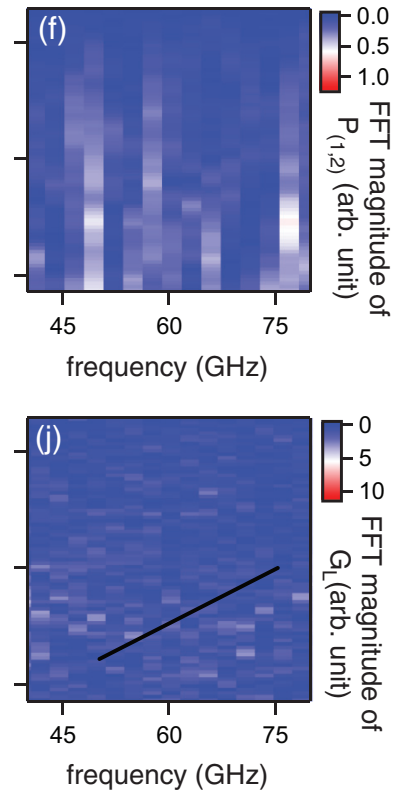
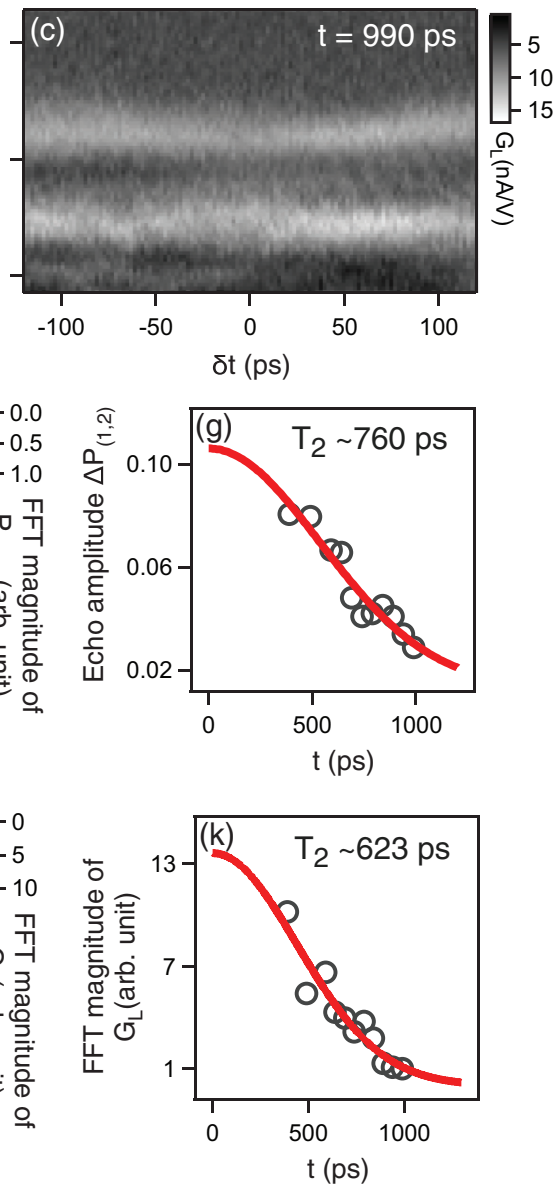

FIG. 4. (Color online) Analysis of echo data for extraction of the decoherence time $T_{2}$. (a)-(c) Transconductance $G_{L}$ as a function of the base level of detuning $\varepsilon_{\mathrm{b}}$ and $\delta t$ (defined in the main text) for total free evolution times of $t=390,690$, and 990 ps, respectively. (d)-(f) Fourier transforms of the charge occupation $P_{(1,2)}$ as a function of detuning $\varepsilon_{\mathrm{b}}$ and oscillation frequency $f$ for the data in (a)-(c), respectively. We obtain $P_{(1,2)}$ (not shown here) by integrating the transconductance data in (a)-(c) and normalizing by noting that the total charge transferred across the polarization line is one electron. Fast Fourier transforming the time-domain data of $P_{(1,2)}$ allows us to quantify the amplitude of the oscillations visible near $\delta t=0$. The oscillations of interest appear as weight in the FFT that moves to higher frequency at more negative detuning (farther from the anticrossing). For an individual detuning energy, the FFT has nonzero weight for a nonzero bandwidth. (g) Echo amplitude as a function of free evolution time $t$. The data points (dark circles) are obtained at $\varepsilon_{\mathrm{b}}=-120 \mu \mathrm{eV}$ by integrating a horizontal line cut of the FFT data over a bandwidth range of 46-72 GHz, then normalizing by the echo oscillation amplitude of the first data point, as described in the text. The echo oscillation amplitudes, plotted for multiple free evolution times, decay with characteristic time $T_{2}$ as the free evolution time $t$ is made longer. By fitting the decay to a Gaussian, we obtain $T_{2}=760 \pm 190$ ps. (h)-(j) Fourier transforms of the transconductance $G_{L}$ as a function of $\varepsilon_{\mathrm{b}}$ and oscillation frequency $f$ for (a)-(c), respectively. As $t$ is increased the magnitude for oscillations at a given frequency decays with characteristic time $T_{2}$. We take the magnitude of the FFT at the point where the central feature (black line) intersects $65 \mathrm{GHz}$. (k) Measured FFT magnitudes at $65 \mathrm{GHz}$ for multiple free evolution times (dark circles) with a Gaussian fit (red line), which yields $T_{2}=620 \pm 140 \mathrm{ps}$, in reasonable agreement with the result shown in $(\mathrm{g})$.

The results presented in Fig. 2 of the main text are obtained by analyzing the oscillation amplitude of $P_{(1,2)}$ at fixed detuning. To get $P_{(1,2)}$ as a function of detuning and $\delta t$, we integrate the time-domain data [such as that shown in Figs. 4(a)-4(c)] from top to bottom. After removing a linear background, we normalize by noting that the total charge transferred across the polarization line is one electron. We perform an FFT (using IGOR $\mathrm{PRO}^{21}$ ), and to ensure that the FFT magnitude is comparable for different values of $t$, we use the same number of points (or equivalently, the same length of time) from each data set by taking a 364 ps cut centered about $\delta t \approx 0$. The transforms are shown in Figs. 4(d)-4(f). The oscillations of interest appear as spectral weight that moves to higher frequency at more negative detuning (farther from the anticrossing). Moreover, for each value of the detuning, the FFT magnitude is nonzero over a certain range of frequencies.

To extract the charge oscillation amplitude from these FFTs, we first take a horizontal trace from the FFT data at $\varepsilon=-120 \mu \mathrm{eV}$, where the pulse tip is around zero detuning. We then integrate the trace over a bandwidth region from 46 to $72 \mathrm{GHz}$. Because for the shortest free evolution time $t$ the oscillations in $P_{(1,2)}$ can be extracted easily from the untransformed data, we use that oscillation amplitude to normalize each of the FFT integrations, allowing us to plot a normalized oscillation amplitude as a function of $t$ in Fig. 4(g). The echo amplitude decays as the free evolution time $t$ is made 
longer, with a characteristic time $T_{2}$. Fitting a Gaussian to the decay yields $T_{2}=0.76 \pm 0.19 \mathrm{~ns}$.

For comparison, we also extract $T_{2}$ from an analysis of the FFT of the unintegrated transconductance data at fixed oscillation frequency. We perform an FFT with a square window function on a $386 \mathrm{ps}$ cut centered at $\delta t \approx 0$ and plot the magnitude as a function of detuning and frequency, as shown in Figs. 4(h)-4(j). We take the magnitude at the point where the central feature (black line) intersects $65 \mathrm{GHz}$ and plot this quantity as a function of $t$ [see Fig. 4(k)]. Fitting to a Gaussian decay yields $T_{2}=0.62 \pm 0.14 \mathrm{~ns}$. As Fig. 4 demonstrates, the two approaches of extracting $T_{2}$ yield similar results.
${ }^{1}$ Y. Nakamura, Y. A. Pashkin, and J. S. Tsai, Nature (London) 398, 786 (1999).

${ }^{2}$ T. Yamamoto, Y. A. Pashkin, O. Astafiev, Y. Nakamura, and J. S. Tsai, Nature (London) 425, 941 (2003).

${ }^{3}$ T. Hayashi, T. Fujisawa, H. D. Cheong, Y. H. Jeong, and Y. Hirayama, Phys. Rev. Lett. 91, 226804 (2003).

${ }^{4}$ K. D. Petersson, J. R. Petta, H. Lu, and A. C. Gossard, Phys. Rev. Lett. 105, 246804 (2010).

${ }^{5}$ K. C. Nowack, F. H. L. Koppens, Y. V. Nazarov, and L. M. K. Vandersypen, Science 318, 1430 (2007).

${ }^{6}$ D. Loss and D. P. DiVincenzo, Phys. Rev. A 57, 120 (1998).

${ }^{7}$ B. E. Kane, Nature (London) 393, 133 (1998).

${ }^{8}$ R. Vrijen, E. Yablonovitch, K. Wang, H. W. Jiang, A. Balandin, V. Roychowdhury, T. Mor, and D. DiVincenzo, Phys. Rev. A 62, 012306 (2000).

${ }^{9}$ D. P. DiVincenzo, D. Bacon, J. Kempe, G. Burkard, and K. B. Whaley, Nature (London) 408, 339 (2000).

${ }^{10}$ Z. Shi, C. B. Simmons, J. R. Prance, J. K. Gamble, T. S. Koh, Y.-P. Shim, X. Hu, D. E. Savage, M. G. Lagally, M. A. Eriksson, M. Friesen, and S. N. Coppersmith, Phys. Rev. Lett. 108, 140503 (2012).

${ }^{11}$ T. S. Koh, J. K. Gamble, M. Friesen, M. A. Eriksson, and S. N. Coppersmith, Phys. Rev. Lett. 109, 250503 (2012).
${ }^{12}$ M. A. Nielsen and I. L. Chuang, Quantum Computation and Quantum Information (Cambridge University Press, Cambridge, 2000).

${ }^{13}$ M. Thalakulam, C. B. Simmons, B. M. Rosemeyer, D. E. Savage, M. G. Lagally, M. Friesen, S. N. Coppersmith, and M. A. Eriksson, Appl. Phys. Lett. 96, 183104 (2010).

${ }^{14}$ C. B. Simmons, J. R. Prance, B. J. Van Bael, T. S. Koh, Z. Shi, D. E. Savage, M. G. Lagally, R. Joynt, M. Friesen, S. N. Coppersmith, and M. A. Eriksson, Phys. Rev. Lett. 106, 156804 (2011).

${ }^{15}$ Z. Shi, C. B. Simmons, J. Prance, J. K. Gamble, M. Friesen, D. E. Savage, M. G. Lagally, S. N. Coppersmith, and M. A. Eriksson, Appl. Phys. Lett. 99, 233108 (2011).

${ }^{16}$ H.-P. Breuer and F. Petruccione, The Theory of Open Quantum Systems (Oxford University Press, New York, 2002).

${ }^{17}$ D. Vion, A. Aassime, A. Cottet, P. Joyez, H. Potheir, C. Urbina, D. Esteve, and M. H. Devoret, Science 296, 886 (2002).

${ }^{18}$ Y. Dovzhenko, J. Stehlik, K. D. Petersson, J. R. Petta, H. Lu, and A. C. Gossard, Phys. Rev. B 84, 161302 (2011).

${ }^{19}$ J. K. Gamble, M. Friesen, S. N. Coppersmith, and X. Hu, Phys. Rev. B 86, 035302 (2012).

${ }^{20}$ Y. Nakamura, Y. A. Pashkin, T. Yamamoto, and J. S. Tsai, Phys. Rev. Lett. 88, 47901 (2002).

${ }^{21}$ www.wavemetrics.com. 